INTERNATIONAL JOURNAL OF SCIENTIFIC RESEARCH

\title{
WANDERING SPLEEN WITH LEFT RENAL AGENESIS - A RARE OCCURRENCE AND DIAGNOSTIC CONUNDRUM
}

\begin{tabular}{l|l|l}
\hline General Surgery & \\
Rankeen Raj* $\quad$ MS (General Surgery), Firozpur, Punjab, India *Corresponding Author
\end{tabular}

\section{ABSTRACT}

The wandering spleen is an unusual condition. It is very rarely associated with renal agenesis. As per the available literature data this is the third such case being reported. The author reports such a case of wandering spleen associated with left renal agenesis, in a ten year old girl who presented with intestinal obstruction.

\section{KEYWORDS}

Wandering spleen; Left renal agenesis; Intestinal obstruction

\section{INTRODUCTION}

Wandering Spleen (WS) is a rare entity. Different terms have been used for this entity such as "displaced spleen," "ectopic spleen," and "dislocated spleen. Wandering spleen is characterized by an excessive mobility and displacement of the spleen from its normal anatomical position. The initial clinical description of the WS is attributed to Van Horne, a Dutch physician, in 1667.(Soleimani et al., 2007) It has an incidence as low as $0.2 \%$ and it is the reason for 2 out of 1000 splenectomies. WS is seven times more common in females than males after age of 10.(Dawson \& Roberts, 1994; Soleimani et al., 2007) The etiology of WS may be multifactorial but it is reported to be associated with renal anomalies like horseshoe kidney(Memari, Nikzad, Nikzad, \& Taherian, 2013; Than, Ten Ern, \& Karim, 2018) and intra-thoracic kidney.(Chappidi, Babu, Pal, \& Srivastav, 2004; Pelizzo et al., 2001) WS along with left renal agenesis has also been very rarely reported in the literature. (Boschert \& Helikson, 1998; Pearson, 1964) The most common presenting sign in adult patients is a mass with or without subacute abdominal or gastrointestinal complaints, or with acute abdominal findings. The most common presentation in children is acute abdominal pain.(Brown, Virgilio, \& Vazquez, 2003; Soleimani et al., 2007) Here the author highlights a case of a child with WS and left renal agenesis presenting as a case of intestinal obstruction.

\section{CASE REPORT}

A 10 year old girl child presented with severe colicky abdominal pain over periumbilical and left lumbar region of one day duration. Her general physical examination was normal. Abdominal examination revealed a distended abdomen, generalised tenderness all over the abdomen and sluggish bowel sounds. A ballotable, non tender, firm lump of $7 \times 5 \mathrm{~cm}$ was palpable in the left lumbar region.

X-ray abdomen erect and supine showed mildly dilated jejunal loops. Ultrasound was doubtful and inconclusive. It showed unascended malrotated left kidney, mildly dilated small bowel loops in umbilical region, absent spleen. Further investigation with CECT of abdomen (Fig.1) revealed signs of sub acute intestinal obstruction, mass in left lumbar region of $76 \times 73 \times 56 \mathrm{~mm}$, absent left kidney and absent spleen.

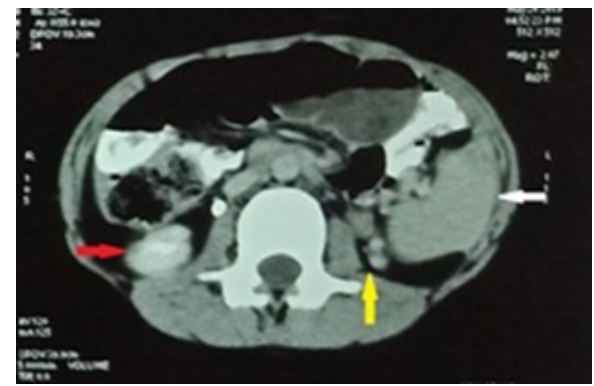

Fig. 1 CECT abdomen showing spleen (white arrow), right kidney (red arrow) and absence of left kidney (yellow arrow)

Patient had progressive abdominal distention, obstipation and absent bowel sounds. Repeat X-ray abdomen showed significantly dilated jejunal loops. She underwent Exploratory laparotomy on Day 2 of admission. Intra-op there were significantly dilated small bowel loops (Fig. 2), normal appearing wandering spleen seen in left lumbar region with its pedicle running across the distal jejunum causing complete bowel obstruction (Fig. 3). The obstruction was released and Splenopexy was done with suture fixation techniques. Post op recovery was complete and was discharged on Day 7.

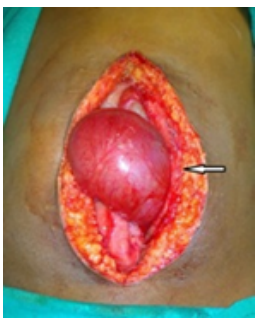

Fig. 2 Intra-operative photograph showing dilated bowel loop

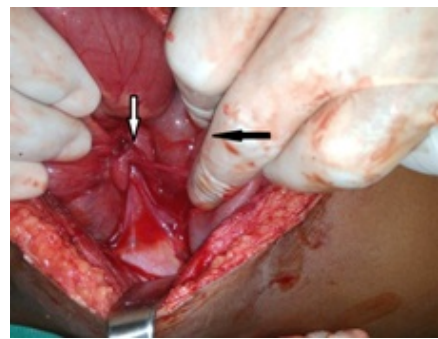

Fig. 3 Intra-operative photograph showing pedicle causing the obstruction (white arrow) and the wandering spleen (black arrow)

\section{DISCUSSION}

WS is a rarely encountered in clinical practise. Even rarer is its occurrence with left renal agenesis. On literature search, it was found that only two similar cases of WS and left renal agenesis were reported in world literature, thus making this finding very rare and worth mentioning. (Boschert \& Helikson, 1998; Pearson, 1964; PRINCE, DUKSTEIN, \& WHITE, 1977)

WS is consequent to the anomalies in the development of the dorsal mesogastrium and the absence or malformation of normal splenic suspensory ligaments, and the mobility of the spleen is dependent on the laxity of the splenic ligaments and blood vessels. It was noted that if the left kidney is absent it increases the mobility of the spleen.(Boschert \& Helikson, 1998) For this reason left renal agenesis will result in increased chance of torsion, when compared to patients with normal left kidney.Clinical diagnosis of WS is challenging. There are reports were WS was confused with Wilms tumor (Pearson, 1964) and seen to be mimicking a kidney on a perfusion study.(PRINCE et al., 1977) In this case also the spleen was confused for left renal mass.

Today the treatment of choice for WS is splenopexy, especially with a healthy spleen and more so for younger patients. Various splenopexy techniques including suturing the spleen to the diaphragm or posterior abdominal wall, by making a peritoneal pocket using native tissue and sutures, applying mesh for fixation by open or laparoscopic techiques.(Soleimani et al., 2007)

WS is a rare entity with varied clinical presentations. It is even rarer to be associated with left renal agenesis. WS in association with left renal agenesis has increased mobility due to absence of lineorenal ligament, hence is at a greater risk for complications. Hence Splenopexy should 
be considered in all cases as the chance of torsion is significantly high.

\section{REFERENCES}

Boschert, M., \& Helikson, M. (1998). Splenic torsion in the presence of renal agenesis. Pediatric surgery international, 13(5-6), 426-427.

2. Brown, C. V., Virgilio, G. R., \& Vazquez, W. D. (2003). Wandering spleen and its complications in children: a case series and review of the literature. Journal of pediatric surgery, 38(11), 1676-1679.

3. Chappidi, S., Babu, V.V., Pal, S., \& Srivastav, D. (2004). Acute mesenteroaxial volvulus associated with congenital diaphragmatic hernia, wandering spleen, and intrathoracic kidney: CT findings. European Journal of Radiology Extra, 52(3), 111-113.

4. Dawson, J. M., \& Roberts, N. (1994). Management of the wandering spleen. Australian Dawson, J. M., \& Roberts, N. (1994). Management
and New Zealand Journal of Surgery, 64(6), 441-444.

5. Memari, M., Nikzad, M., Nikzad, H., \& Taherian, A. (2013). Wandering spleen in an adult man associated with the horseshoe kidney. Archives of Trauma Research, 2(3), 129.

6. Pearson, J. (1964). Torsion of the spleen associated with congenital absence of the left kidney. British Journal of Surgery, 51(5), 393-395.

7. Pelizzo, G., Lembo, M., Franchella, A., Giombi, A., D'agostino, F., \& Sala, S. (2001). Gastric volvulus associated with congenital diaphragmatic hernia, wandering spleen, and intrathoracic left kidney: CT findings. Abdominal imaging, 26(3), 306-308.

8. PRINCE, J. R DUKSTEIN, W. G., \& WHITE, W. E (1977). A Wandering Spleen Which Mimics a Kidney on a Perfusion Study. Clinical Nuclear Medicine, 2(8), 274Which Mimics a Kidney on a Perfusion Study. Clinical Nuclear Medicine, 2(8), $274-$ 275.

9. Soleimani, M., Mehrabi, A., Kashfi, A., Fonouni, H., Büchler, M. W., \& Kraus, T. W. (2007). Surgical treatment of patients with wandering spleen: report of six cases with a review of the literature. Surgery today, 37(3), 261-269.

10. Than, D. J., Ten Ern, T., \& Karim, K. B. A. (2018). Wandering spleen with horseshoe kidney a rare occurrence. International journal of surgery case reports, 45, 96-100. 